\title{
Bubble NoC - A Low Energy Network-on-Chip with Small Footprint and High Performance
}

This paper was downloaded from TechRxiv (https://www.techrxiv.org).

\section{LICENSE}

CC BY-NC-SA 4.0

SUBMISSION DATE / POSTED DATE

$15-12-2021 / 17-12-2021$

\section{CITATION}

Carlstedt, Gunnar; Rimborg, Mats (2021): Bubble NoC - A Low Energy Network-on-Chip with Small Footprint and High Performance. TechRxiv. Preprint. https://doi.org/10.36227/techrxiv.17206295.v1

DOI

10.36227/techrxiv.17206295.v1 


\title{
Bubble NoC - A Low Energy Network-on-Chip with Small Footprint and High Performance
}

\author{
Gunnar Carlstedt and Mats Rimborg
}

\begin{abstract}
The Bubble NoC is based on simplicity and provides outstanding performance. Flow control is implemented by bubbles, which are inserted between the flits. The logic resembles a traffic situation where a vehicle only moves if the next position is empty. When a flit moves, a bubble is created behind it, and when there is a blocking the bubbles are collapsed as the flits behind are packed together. Even when the Bubble NoC is saturated, it degrades gracefully, and the execution continues.

Deterministic prerouting is used, with the address stored as markers in a 2 out of 32 code. The routing algorithm shifts the address one step at each hop and turns or finishes when a marker starts the address.

The physical implementation is a mesh of streets containing duplex links of 38 wires carrying 32-bit payload. Signaling is based on current injection that charges the wires. A switch is placed in a four-way crossing, with a fifth local connection into a street. The switch contains input registers for each approaching street. Straight through traffic is simply passed on, and a diagonal gate is used for turning traffic.

All switches are bidirectional transmission gates, and the control is distributed as a sidewalk in a few $\mu \mathrm{m}$ of the periphery surrounding the intersection. In a $14 \mathrm{~nm}$ technology, the streets are $8 \mu \mathrm{m}$ wide, the crossing is $17 \mu \mathrm{m}$ in square, the hop frequency 6.67 GHz and the energy for a datapath $4.1 \mathrm{fJ} / \mathrm{bit} / \mathrm{hop}(150 \mu \mathrm{m})$.
\end{abstract}

Index Terms-bufferless network, current injection, flow control, low energy, network-on-chip

\section{INTRODUCTION}

$\mathrm{W}$ ith Moore's Law approaching its end due to physical realities, sustainable future improvements of computing must depend on scalable multiprocessors. These should be based on planar silicon technology and come in different sizes and shapes, from sub-mm chips to stacks of wafers. The processors of tomorrow will contain arrays of processing elements (PE), optimized for parallel execution rather than single-core performance.

We anticipate small, simple PEs with sides as short as a few thousand wire pitches $(100 \mu \mathrm{m}$ in a $14 \mathrm{~nm}$ technology). These devices can be separated by lanes for a mesh network-on-chip (NoC). Such networks need to be small and consume low energy, compared to the PEs. It will be hard to implement a reliable software-based delivery, due to the vast number of

This paragraph of the first footnote will contain the date on which you submitted your paper for review.

The authors are with the Department of Computer Science and Engineering, Chalmers University of Technology, SE-412 96 Göteborg, Sweden (e-mail: gunnar@carlstedt.se and mats@rimborg.se). parallel processes and large resulting traffic. Power and speed will be important, as they always have been.

The Surface Base Processor (SBP) is a new emerging such computer architecture [1]. The SBP has the potential to contain hundreds of thousands of nodes, thus providing a huge computing capability but also requiring an extremely high communication bandwidth. It may have a random communication pattern from computations that can saturate a network. To our knowledge, no NoC able to satisfy this has been published.

Simplicity is a key to the solution. A low-level, deadlockfree protocol is needed, with very simple flow control and guaranteed deliveries. It should work under all load conditions and degrade gracefully in a saturated network. The physical implementation of a low-voltage high-speed link is one part of the solution. The highest priority has been to achieve a small area, then low energy, and finally high speed.

In this article, such a network is presented. It has been studied by Spice simulations and modeled in software. The payload of flits are 32 bits wide, matching the intended hardware but not architecturally limited to this, and transported with a $150 \mathrm{ps}$ cycle time.

The main contribution is:

- Four logically separate networks are implemented as shared pipelined wires. Routes are monotonic in both $\mathrm{x}$ and y-directions.

- A deterministic flow control using bubbles is used within each network. Delivery and graceful degradation are guaranteed, even when the network is saturated.

- Routing is performed using a linear address counter.

- Current injection of wires through switches drive the transmission lines.

- The switch forms a small and energy efficient crossbar.

- A network, almost as narrow as its wires, surrounds the PE devices.

The remainder of this article is structured in the following way. Section II is an overview of the design. In section III, the internal communication is discussed. A short overview of various options for the routes is made in section $I V$. The flow control mechanism is covered in section $V$.

The network node and routing control are described in section VI. Sections VII and VIII are focused on the hardware and how to achieve low energy consumption.

Finally, section $I X$ describes the NoC evolution and compares the Bubble NoC to current state-of-the-art, while section $X$ contains our conclusions. 


\section{OVERVIEW}

\section{A. Structure}

A mesh network consists of rectangular devices surrounded by wire lanes. In the corners, where the lanes intersect, network nodes (switches) are placed, see Fig. 1. The network node is outlined in section VIII, and shown in Fig. 14.

\section{B. Wire Lanes and Cross Talk}

A lane contains a bundle of wires close to each other, with 32 wires carrying the payload and a 5-wire tag, see Fig. 1, and there is a ground plane with fixed potential. Each wire interferes with two neighbors and the ground plane. There are capacitances between all of these that can be described as a ladder of $\pi$-stages. Each such step has a series capacitance $C s$ and two ground capacitances $\mathrm{Cg}$. A signal in one wire is induced into the neighboring wire. If both wires on each side carry the same signal, they induce the same signal level in the affected wire when the attenuation is $1 / 3$ [2].

A driver adds a resistance of a transistor in parallel to $C g$, with a typical equivalent resistance of $50 \mathrm{k} \Omega$. The wire segment has the capacitance $20 \mathrm{fF}$ providing a time constant of $1 \mathrm{~ns}$. This is too high to reduce the disturbing signals.

It is possible to get an undisturbed transmission by using opposite polarities of a Zero and One when the attenuation is less than $1 / 3$, and this is a requirement on the conductor cross section. It can be achieved in many ways, e.g., by using every second wire as ground [3].

This requirement implies a wire lane width of $6 \mu \mathrm{m}$. Near the corners an additional $2-3 \mu \mathrm{m}$ are needed for the driver stage to the network node.

\section{Signal}

A wire is mainly capacitive, and a small standard transistor may drive it with $28 \mu \mathrm{A}$. During $75 \mathrm{ps}$, the signal rises to $70 \mathrm{mV}$ in a somewhat distorted way. The signal may be coded as two ramps caused by currents: Zero $\{-\mathrm{i},+\mathrm{i}\}$ and One $\{+i,-i\}$, see Fig. 2. In both cases, the voltage level is restored after a full cycle.

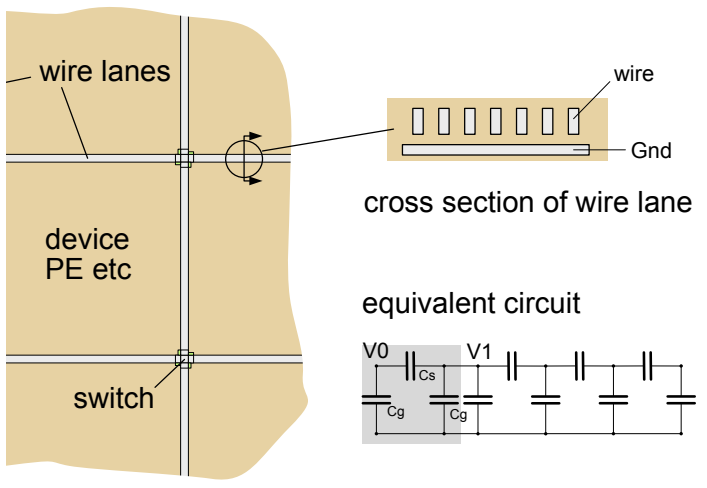

Fig. 1. A mesh of devices with surrounding wire lanes.

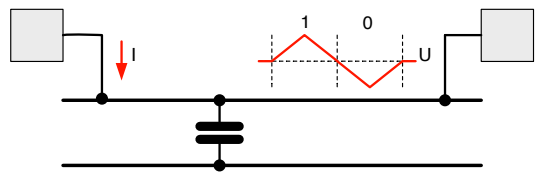

Fig. 2. Current injection of capacitive wire.
If the wire has a series resistance $r s$ and capacitance $c s$ per length $l$, the time constant will be $r s \times c s \times l^{2}$. Metal layers can be very different, e.g., M1 135 ps and M3 6.8 ps. The responses at different frequencies are shown in Fig. 3. The signal level is very low in the first case. The symmetric current sequences make both signals detectable, however, with different delays.

Current is a perfect signal carrier to switch with transmission gates and can be fed into a long wire for use in the network node, see Fig. 10. The source may be located anywhere along the wire. It is used to connect the local device to a network node. There are other low voltage implementations [4], however, they don't fit due to speed, high capacitance, and low current. Current mode is not discussed in this article.

The signal has to be detected by a sense amplifier, and the same technique is used as by DRAMs. These amplifiers are small and may be placed under a wire, see Fig. 15.

\section{Pipelined Wire and Full Duplex}

A pipelined wire is divided into segments surrounded by master-slave flip-flops, see Fig. 4. Except for the flip-flops, the propagation delay and the energy consumption are independent of the number of segments. The switched charge in each segment is reduced proportionally, and the transport capacity increases.

Two such oppositely directed pipelines may be placed in parallel for duplex operation, Fig. 4c. The wires may also be shared to save area, Fig. 4d. Such pipelines use time-division duplexing, where the transport capacity is shared between the directions. The propagation speed is therefore halved.

In a two-dimensional mesh structure, each pipelined wire has its own register in a node, i.e., a total of four registers. The pipelines act independently, so no blocking or contention will occur.

A pipelined wire makes use of the two phases to evaluate and transport. During the first phase, the conditions are decoded and used to set up the switches for the next phase.

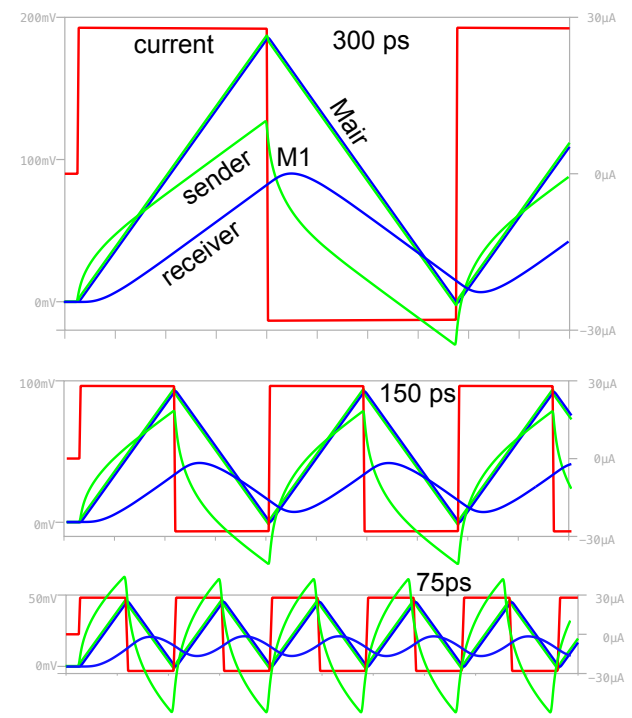

Fig. 3. Signal frequency response and dependence on wire time constant. 
(a)

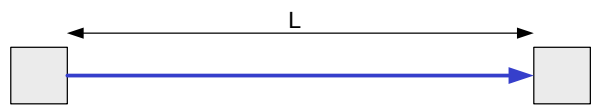

(b)

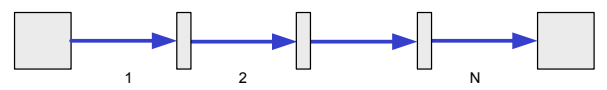

(c)

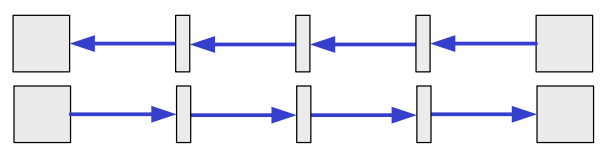

(d)

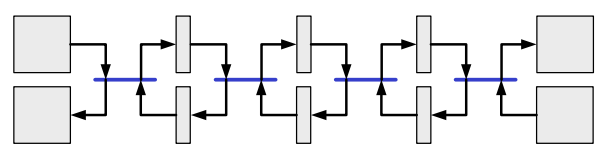

Fig. 4. Transmission lines. From top to bottom: (a) wire, (b) pipelined wire, (c) separate duplex wires, and (d) duplex implemented with shared wires.

\section{E. Deadlock Free Virtual Channels}

Deadlock is generally prevented by using virtual channels. Here, four networks NW, NE, SE, and SW are used. The propagation is monotonic and therefore deadlock free [5], see section III. They are implemented by joining one horizontal W-E and one vertical S-N pipelined wire, see Fig. 6. A flit is injected horizontally and turned into a vertical pipelined wire, and both wires may use either forward or backward phase. Therefore, a turn may imply a phase shift. An additional turn buffer register is used to bridge the additional needed cycle.

\section{F. Flow Control}

The flow control is implemented by introducing an additional value, called a bubble, which indicates an empty position. The algorithm resembles a traffic situation where a vehicle only moves if the next position is empty, see Fig. 7. It may be a straight forward movement or a turn. The first vehicle moves and creates a bubble behind it, then the next one follows, and so on. Correspondingly, packing occurs when there is a blocking, see section $V$.

This is implemented by sending bubbles upstream. When moving, all flits move at full speed. However, the message length has doubled because of the bubbles.

The mechanism is much simpler than conventional bufferless NoCs [6]. Also, bufferless methods cannot guarantee deliveries.

\section{G. Routing and Addressing}

Rerouting uses energy, and in some cases a large amount of energy. A router limits the speed and uses a considerable area. Instead, deterministic prerouting is used, see Fig. 5. An address that consists of direction (NW, NE, SE, or SW), $d x$, and $d x+d y$ is used.

Within the message, the address represents the remaining part of the route. The direction is coded by the implied position (east-west/north-south), phase (forward/backward), and a bit turn left/right. The $d x$ and $d x+d y$ are counters implemented in linear form by marking a position in a shift register. At each hop, the register shifts left and the positions for $d x$ and $d x+d y$ accordingly decrease. An action takes place when there is a marker in the first position, otherwise there is a straight shift, see section $I V$.

\section{H. Switching}

Switching means simply to connect the pipelined wires. The switch structure is shown in Fig. 10. There are 16 switch cases, see Fig. 11. Ten of them engage the entire switch, while there are six (named straight) of which two may occur in parallel in the $\mathrm{x}$ - and y-directions, see section VI.

The switch contains a register in a pipelined wire for each direction, two turn buffers, and several switches. Among them, there is one bend that shifts from the $\mathrm{x}$ - to the $\mathrm{y}$ direction. The implementation maps this structure in the crossing of the wire lanes, see Fig. 14 and section VIII.

The energy for one wire that is used in the sense amplifier, including the register and the switch, is $0.63 \mathrm{fJ} /$ wire, driving the datapath requires $0.65 \mathrm{fJ} /$ wire, and the energy lost in the wire is around $1.0 \mathrm{fJ} /$ wire. In total, including small miscellaneous sources, the energy per payload bit is $2.56 \mathrm{fJ} / \mathrm{bit}$. When including the control unit, the energy consumption is $4.1 \mathrm{fJ} / \mathrm{bit} / \mathrm{hop}$, see section VIII.A. The switch is very simple compared to those discussed in the literature.

\section{Control}

The control is divided into 16 cases. Each pipelined wire has several AND-gates indicating a state of what to do. These build the corresponding condition using status from the other pipelined wires. A priority decoder selects a case and produces control signals for each of the pipelined wires.

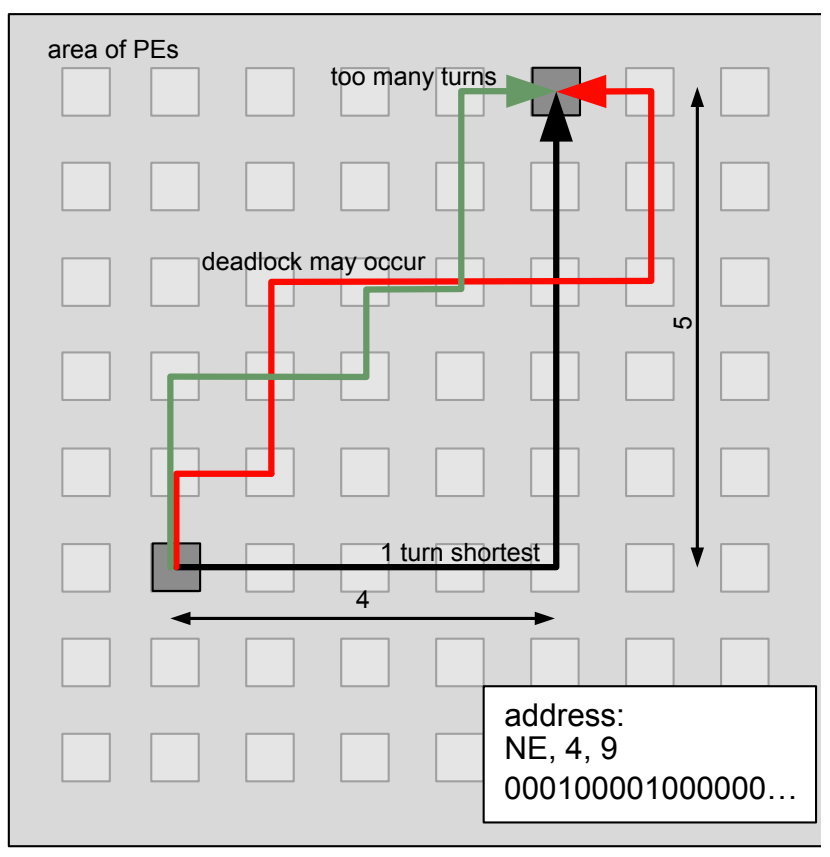

Fig. 5. Routes; improper (red and green) and good (black). 


\section{SOURCE AND SINK}

A PE communicates with other PEs, sending messages as packets of several words. On the message level, deadlock, livelock, and starvation should be avoided. This means that a receiving PE should act as a sink and absorb all messages, see Fig. 6.

In a mesh, there are links between nodes and PEs. Thus:

- a node sending a packet to its local PE is deadlock-free, living, and not starving. Packets are resorbed at high frequency.

- for an outgoing link of a node, there are three incoming links. When the output is unblocked, so are the inputs. Thus, a node next to the node mentioned in the previous paragraph is deadlock-free. Input ports are living and not starving as long as the arbitration is fair. If turning routes have lower priority, the straight direction (along the $y$ axis in Fig. 6) is not starving.

- a node may have an output to another node. Recursively, the earlier condition applies as long as the links are monotonously followed along the $\mathrm{x}$ - and $\mathrm{y}$-axes, i.e., there is a steadfast movement towards NW, NE, SE, or SW.

- a PE is connected to a node. If injection has lowest priority, packets traveling along the $\mathrm{x}$-axis are living and do not starve. Because no nodes along the $\mathrm{x}$-axis are starving, input packets are guaranteed to move forward. PE's backstream along the $\mathrm{x}$-axis may suffer from starvation. Therefore, the injection has to be fair.

The third case may cause a deadlock. However, the links are full duplex, and the route is not allowed to turn back: A path starts by design in the $\mathrm{x}$-direction; it may turn left or right; then the path continues in the y-direction. There are no turns from the y-direction, so a loop cannot be formed.

The fourth case may cause starvation. A PE continuously injecting packets into the network may occur. However, this is a case that a compiler may eliminate. In practice, this situation can always be avoided by throttling the PE stream. Therefore, no global mechanism for fair injection is necessary.

Thus, deadlock-free, live paths, and no starving PEs, can be guaranteed.

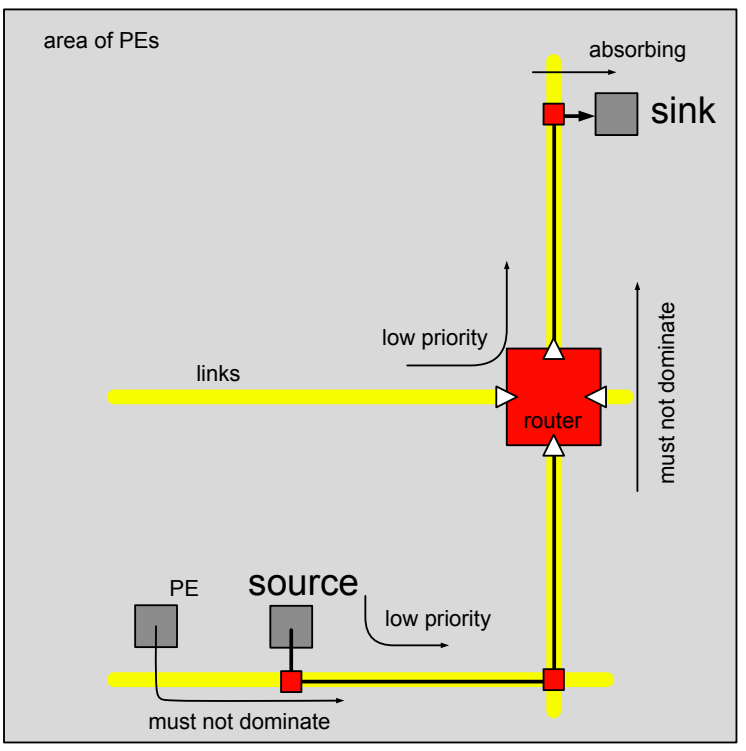

Fig. 6. Source and sink.

\section{ROUTES}

There is a route from source to destination, see Fig. 5. It is shortest when it is monotonic in both $\mathrm{x}$ - and $\mathrm{y}$-directions. Then the number of passed nodes and links are independent of the selected route. The consumption of wire energy is proportional to the number of links. The route may in some cases be between two PEs that have the same $\mathrm{x}$ - or $\mathrm{y}$-coordinates, with no turns. Other routes may have several turns. Each such turn must either be stored in the message or calculated. Both methods will impair the power consumption. Therefore, there should be only one turn [7]. The energy is fixed and proportional to the route length.

\section{FlOw CONTROL}

The pipelined wires have registers between each link. These registers can be used as storage. The algorithm is simple: if the next register is free then transport, otherwise wait, see Fig. 7. Queued flits are packed together along a route. Transport is started by the forwardmost flit, which creates a free register, the bubble. The next register transports to the bubble, and so it continues. Bubbles flow backstream along the registers and create new bubbles between the flits. The flits with bubbles move forward at full speed. At a stop, bubbles will be lost, and the flits packed together.

This method is fully reliable, however, during transports every second flit is a bubble. Each flit has a tag free/used, which is the only bit used for the flow control. The circuit is simple and allows single-cycle propagation of non-waiting flits.

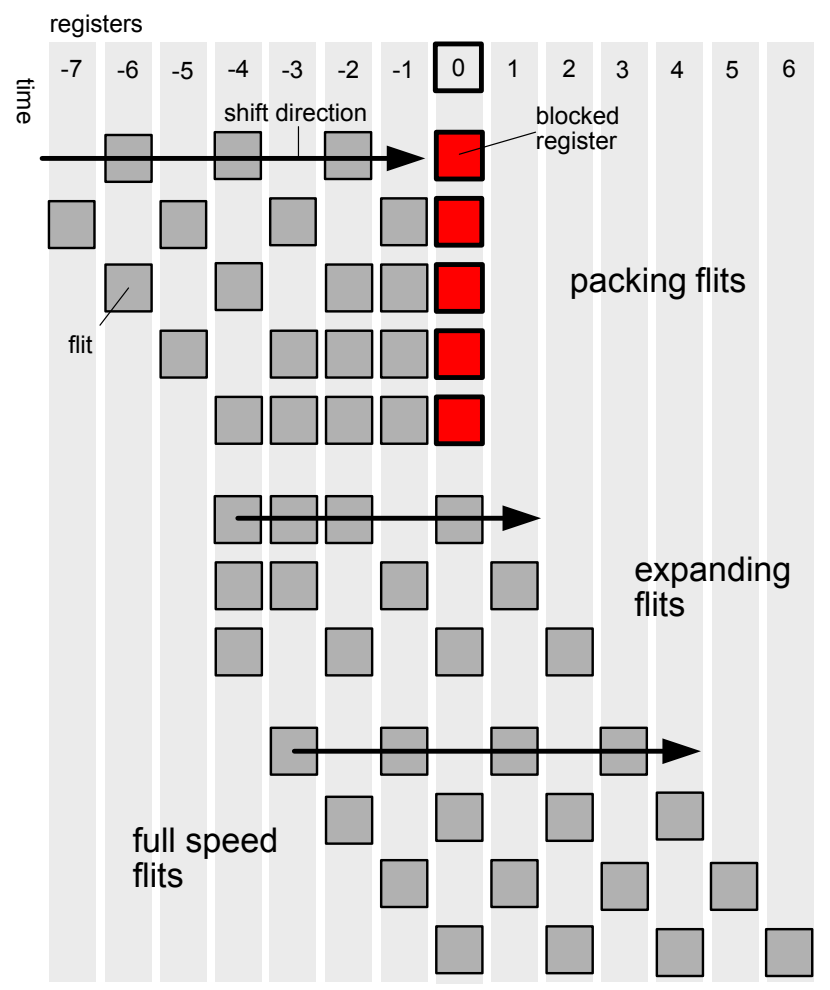

Fig. 7. Moving bubble. 


\section{A. Flow Control Algorithm}

A flit may be moved forward if the node ahead is empty. This resembles a line of people/cars in a queue. If every second position is free, the queue may move with arbitrary speed. If not, the first flit in the queue moves and creates a space. It continues until there is space between all used positions.

To implement this, for each word $\mathrm{v}[\mathrm{k}]$ of the mesh pipeline, two tags $U[k]$ and $U_{n}[k]$ are added, see Fig. 8. Moves are performed in increasing $\mathrm{k}$ direction. The $\mathrm{U}$ (used) tag indicates that the word is not empty. $U_{n}[k]$ indicates the same for the next forward word $\mathrm{U}[\mathrm{k}+1]$. A word is moved forward together with its $U$ tag. The $U_{n}$ tag is moved in the other direction. If the $U_{n}$ is free and $U$ is used, the current word $v$ is moved forward and $U$ and $U_{n}$ are set free and used, respectively. Otherwise, $\mathrm{U}_{\mathrm{n}}[\mathrm{k}]$ is set to $\mathrm{U}_{\mathrm{n}}[\mathrm{k}+1]$.

In a routing node, the $U_{n}$ ahead needs to be used for all possible routing directions. There are three such ways: left, straight and right. For each direction, the $U_{n}$ has to be selected according to the current flit.

\section{B. Long Packets - Worms}

The payload length is at most 32 words, which are transported by wormhole routing. Flits are tagged payload/first. Routing decisions are made for the first flit and applied to the entire packet.

There is no reassembly of packets, or messages. The control of a packet lasts from the detected tag in the first flit and is kept in a register until the last flit of the packet has passed.

The control is divided into three processes for each forward and backward direction: a turn-, an x-, and a y-process. A tag initiates a turn-process. It waits until the $\mathrm{x}$ - and $\mathrm{y}$-processes are finished. The $\mathrm{x}-$ and $\mathrm{y}$-processes act separately. They can be initialized when the turn-process is idle. See Fig. 9.

\section{NeTWORK NoDE}

The traffic is shifted forward and backward along the $\mathrm{x}$ - and $y$-axes, depending on the direction of the route. Some traffic

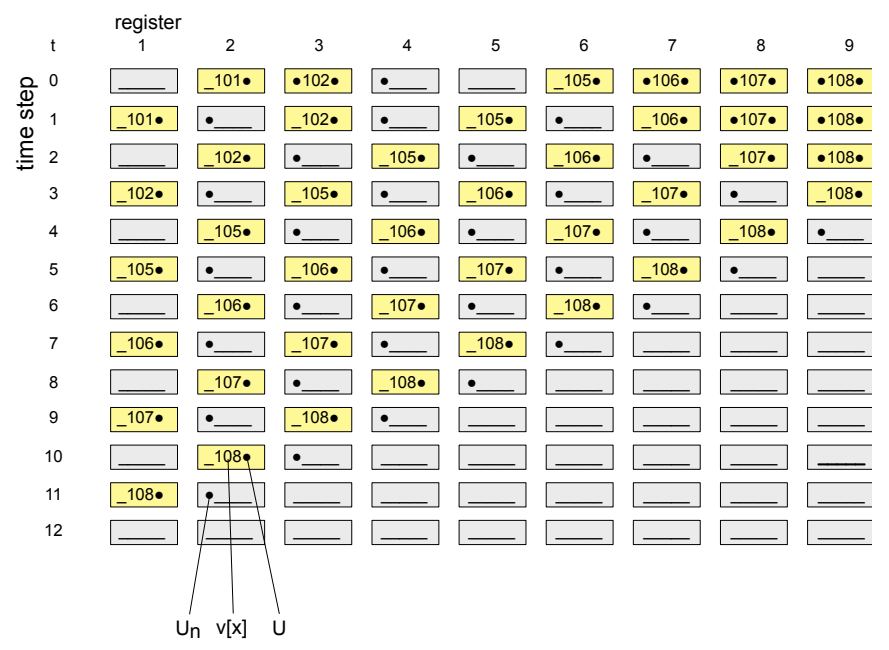

Fig. 8. Link with word moving in the backward direction (to the left) while bubbles move forward (to the right). passes a node while other traffic turns at a node. In both cases, a shift register implementation is used. During each cycle the traffic moves, forward or backward. If no turn takes place, the traffic may pass a node in both $\mathrm{x}$ - and $\mathrm{y}$-directions simultaneously. If a turn takes place, only the turn is performed. One could argue that a second non-overlapping turn could be performed. This is opted out, because it would increase the switch size considerably. Turning occurs much more seldom than passing straight through since no route needs to have more than one turn.

Each network node is a router, placed in the junction of links in the $\mathrm{x}$ - and $\mathrm{y}$-directions. There is a communication channel in each direction; E, N, W and S, see Fig. 10. There are also two channels, L, connecting to neighboring PEs, see below for a more detailed description. Each route contains at most one turn, and the route lengths are generally several hops. Therefore, the most common case is packets passing straight through the nodes. Different ways to implement the turns cause varying complexity.

When forwarding packets, each of the four communication channels has three possible inputs. There is an input from the opposite direction for messages that will pass straight through the node, and two for the bends. Additionally, the L-channels may be the destination for packets from any direction, and the source for packets exiting the node to $\mathrm{E}$ or $\mathrm{W}$ (possibly immediately taking a turn in the next node). In total, there are six inputs. They allow two straight or two turns at the same time. All combinations are seldom needed simultaneously. The wires and the transistors for the turns are somewhat complex. To simplify, only two simultaneous straight through routes and one turn from the $\mathrm{x}$-direction are implemented, see Fig. 11.

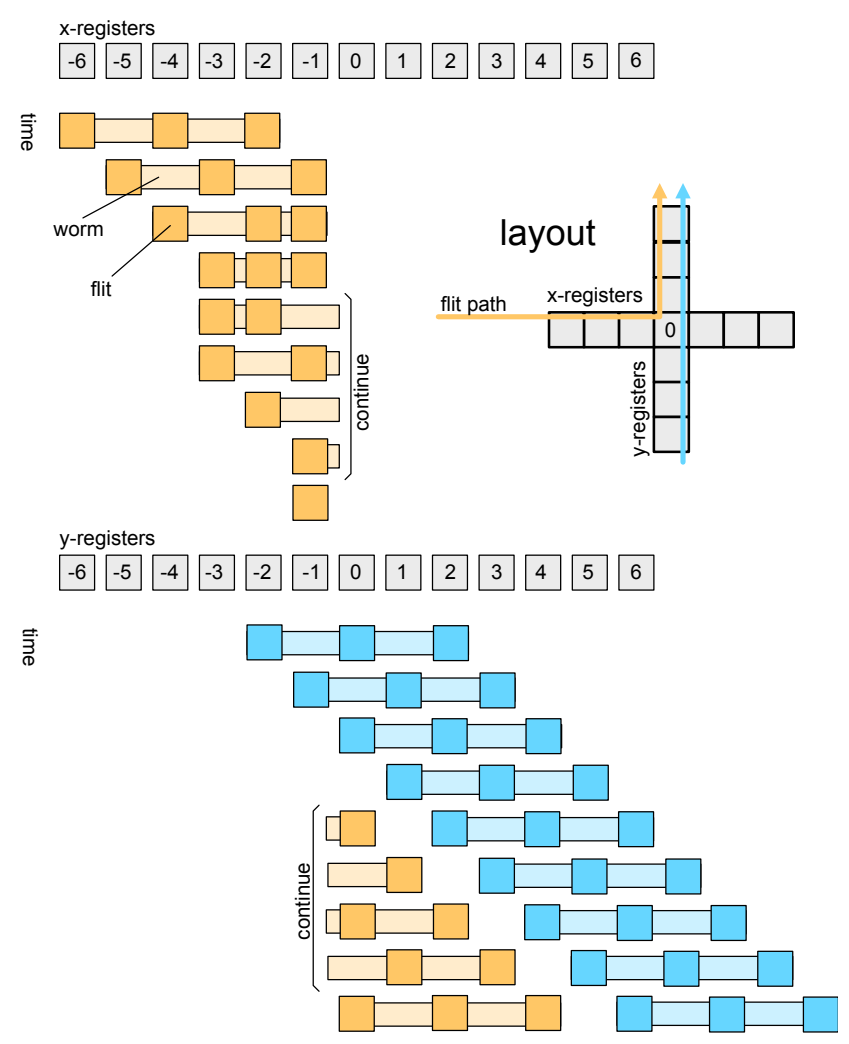

Fig. 9. Worm flow control. 


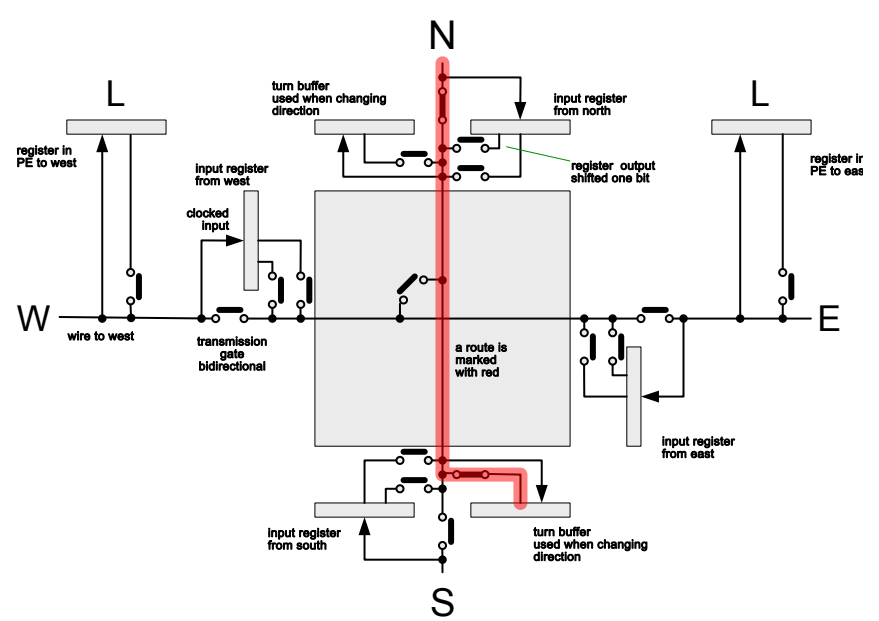

Fig. 10. Network node.

The straight routes are uncomplicated. A turning route in forward/backward direction may switch to the other direction backward/forward and use the other phase. To solve this, a buffer register is used to transfer data between the phases. The buffer is placed as an auxiliary register in the new opposite direction. Buffers are only needed in the north and south directions, since transports along the $\mathrm{x}$-axis are always performed first. The bend is to the input in the opposite direction. During next phase, transmission is performed as a straight transfer, but from the buffer.

\section{A. Connection to PE}

Typical mesh networks use nodes with five connections: E, $\mathrm{N}, \mathrm{W}, \mathrm{S}$, and $\mathrm{L}$, where $\mathrm{L}$ is the link to the local PE [8]. In the network described here, the connection is somewhat changed due to layout considerations. The network consists mainly of narrow wire lanes. Using a node with five inputs would change the simple layout considerably. Instead, the PE is connected to the wires between two nodes along the x-axis. The PE may receive or send messages in both the forward and backward directions along the $\mathrm{x}$-axis.

\section{B. Routing Control}

Routes are performed in one of four directions as earlier described: NW, NE, SE, and SW. The distances to the turn and to the destination are coded in the first word of a message. It is a linear mapping of the distances; all bits are zero except for one or two, specifying the turn (if there is one), and target positions. For each node a message passes by, the first word is shifted left and padded with ones, thereby efficiently extending the width to 33 bits. When there is a transport in the $\mathrm{x}$-direction, a 1 in the first position indicates a turn. The destination is reached when transporting in y-direction and a 1 is in the first position. The address may specify up to 32 hops.

A word in the mesh pipeline has an indication used $\mathrm{U}$ (1 bit), next used $\mathrm{U}_{\mathrm{n}}(1 \mathrm{bit})$, first flit (1 bit), a turn direction left, right and target on $\mathrm{x}$-axis (2 bits) of a message. Together with the direction phase, they control the action of the node.
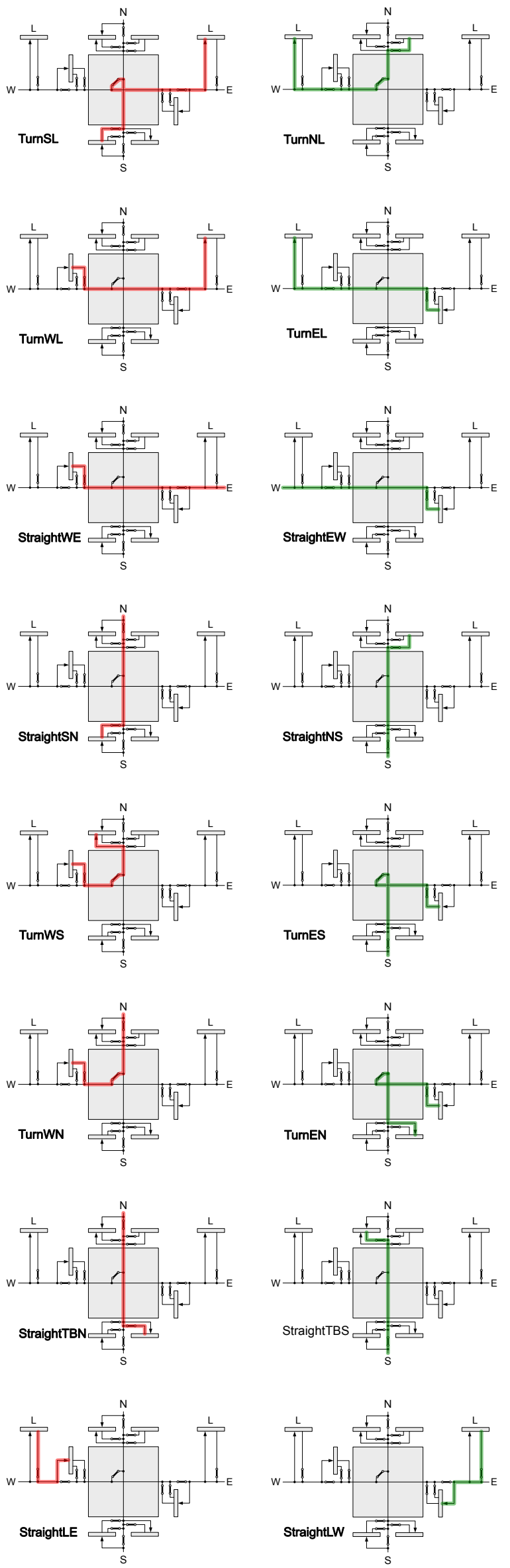

Fig. 11. Route semantics. Red paths indicate forward direction in phase one, green paths indicate backward direction in phase two. 
There are 16 possible operations, of which the two pairs that use turn buffers are always performed subsequently, and 10 of them are turns, see Fig. 11. For each of the operations, it is decoded in an input buffer, the flow control is applied, and a request is calculated for the operation. The operations are scheduled in priority order, 1 is highest.

1. an absorb operation sending a message into a PE (4 cases; TurnSL, TurnNL, TurnWL, TurnEL)

2. a straight operation passing through the node (4 cases, one for each direction; StraightWE, StraightEW, StraightSN, StraightNS)

3. a turn operation utilizing both the $\mathrm{x}$ - and y-directional mesh pipelines (4 cases, of which two of them use a turn buffer to shift phase; TurnWS+StraightTBS, TurnES, TurnWN, TurnEN+StraightTBN)

4. an inject straight operation where a PE injects a word in an $\mathrm{x}$ mesh pipeline ( 2 cases; TurnLE, TurnLW)

Control signals are generated for each case in a Finite State Machine (FSM). The control is individual for each phase. In a forward phase, flits on the $\mathrm{x}$ - and $\mathrm{y}$-axes are moved forward and written to an input register. During the next phase, the input registers are decoded, scheduling is performed, and control signals are generated. In the following phase, the control signals are used when sending flits. The corresponding actions are performed in the backward direction.

The node control algorithm for the FSM has been modeled in software and verified to function correctly.

\section{LOW-ENERGY WIRE}

The schematics of a link is shown in Fig. 12a. It consists of registers separated by a wire. The wire is driven by a current from a register. The wire is interrupted by transmission gates (not shown). At the receiver end, a flip-flop is used as sense amplifier. With an additional flip-flop, a master-slave register is formed. Because of the low switching voltage, the transmission gates do not need to be complementary.

A clock using an initial phase after the rising edge controls the link, see Fig. 12b. During phase one, the transmission-gate $\mathrm{M} 3+\mathrm{M} 4$ is not conducting. The transmission-gate M5+M6 discharges the wire to an intermediate voltage $V i$. During the second phase, the driver is stable and the transmission-gate $\mathrm{M} 3+\mathrm{M} 4$ is conducting. The wire is charged, and the receiver flip-flop is unpowered. Its node Qsense is connected to the wire by transmission-gate M7. The other node Qsensb is connected via the transmission-gate M14 to an intermediate voltage $V i$. In the next phase, the transistors M10 and M11 of the receiver flip-flop conduct and form a dynamic sense amplifier. Initially, the node Qsense has the voltage of the link wire. The node Qsense switches towards VDD or GND.

Transistors M15 to M24 form a slave register. Transistors M15 to M18 set the register in the next clock phase. Transistors M19 to M22 make the feedback in a following clock phase.

Transmission-gate M3+M4 consists of several serial coupled transistors used by the routing mechanism. The conducted current is low, generally several times less than for conventional transistors. The switching voltage on the wire is a sawtooth. Its amplitude depends on the current, the phase duration, and the wire capacitance. The receiving flip-flop is isolated when switched, and only its own capacitances are charged. Their energy is low compared to the wire energy. During the transition period of some ps, the flip-flop is short circuited, but the energy is negligible.

A mesh pipeline is full duplex, thus a transport on a wire is performed with half frequency. The other cycle is used for the opposite direction, so there is hardly any loss of throughput.

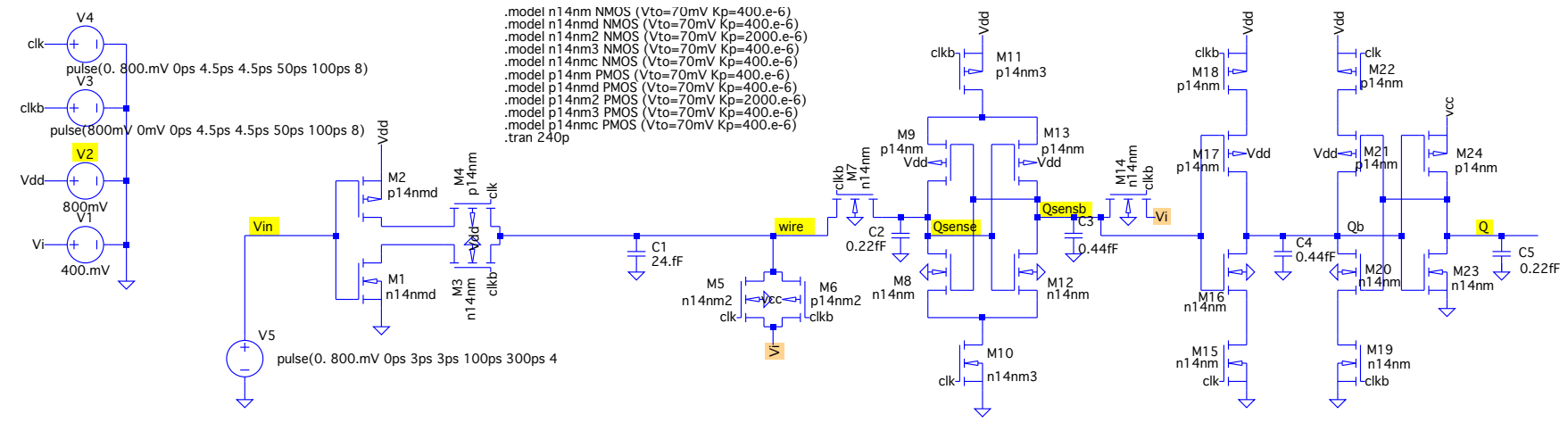

(a) Circuit diagram of a link section in a pipelined wire.

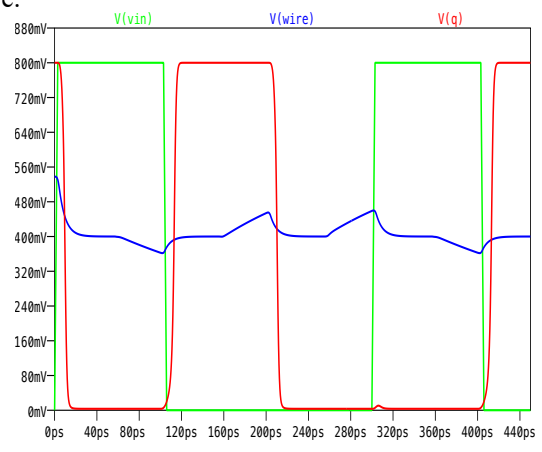

(b) Delays in the pipelined wire.

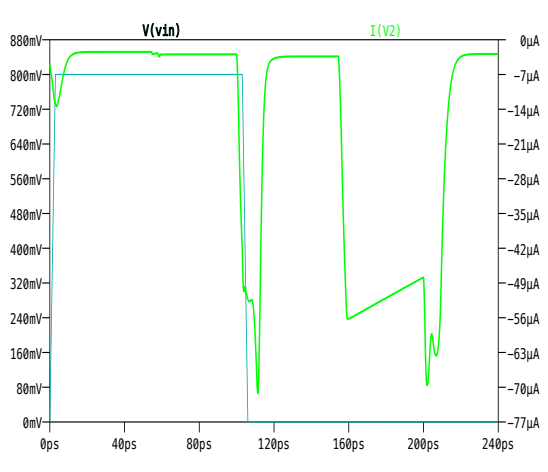

(c) Current in the pipelined wire.
Fig. 12. Pipelined wire. 


\section{A. Spice Simulation of the Wire}

Transistor and wire characteristics are chosen for a $14 \mathrm{~nm}$ technology [9], [10], [11]. There is a series of waveforms Vin, wire and $Q$, see Fig. $12 \mathrm{~b}$. The propagation delay from clock clk to the output $Q$ is $9.7 \mathrm{ps}$. The current injection is around $\pm 25 \mu \mathrm{A}$. There are spikes in the waveform for the transitions. The charge and energy per cycle at $800 \mathrm{mV}$ swing are $2.0 \mathrm{fC}$ and $1.6 \mathrm{fJ}$.

\section{THE SWITCH}

Four low-energy wires implement the network node, see Figs. 12 and 13. Only the south wire is shown in Fig. 13, and the control unit is also omitted. Transistors MS5 to MS24 implement the pipelined wire in the south direction. MW23 and MW24 form the output stage of the slave register in the west pipelined wire. Transistors MW25 to MW30 indicate optional switches used in any direction, placed here during simulation, see Fig. 10.
Transistors MT1 and MT2 form the transmission gate in the middle of the switch. $W$ and $S$ are internal wires to the switch. The link wires are Wwire and Swire.

\section{A. Spice Simulation of the Switch Datapath}

There is a series of waveforms $Q W, Q S$ and $Q$, see Fig. $13 \mathrm{~b}$. The current fed into the wires creates a sawtooth voltage and is strobed by the sense amplifier and stored in the slave latch, see Fig. 13c. The current from $V_{D D}$ is shown in Fig. 13d. The shape resembles the one in Fig. 12c.

The transmission gates may be implemented in CMOS or with n-channel transistors. The number of wires and transistors are almost halved in the second case compared to the first case.

The sawtooth voltage for the CMOS case is reduced from the input $Q W 726 \mathrm{mV}, W 648 \mathrm{mV}, S 573 \mathrm{mV}$ and finally to Swire $497 \mathrm{mV}$, see Fig. 13c. The injected current is around $30 \mu \mathrm{A}$. The n-channel case has lower voltages; $778 \mathrm{mV}$, $550 \mathrm{mV}, 472 \mathrm{mV}$ and $425 \mathrm{mV}$ when $7 \mu \mathrm{A}$ is injected. By

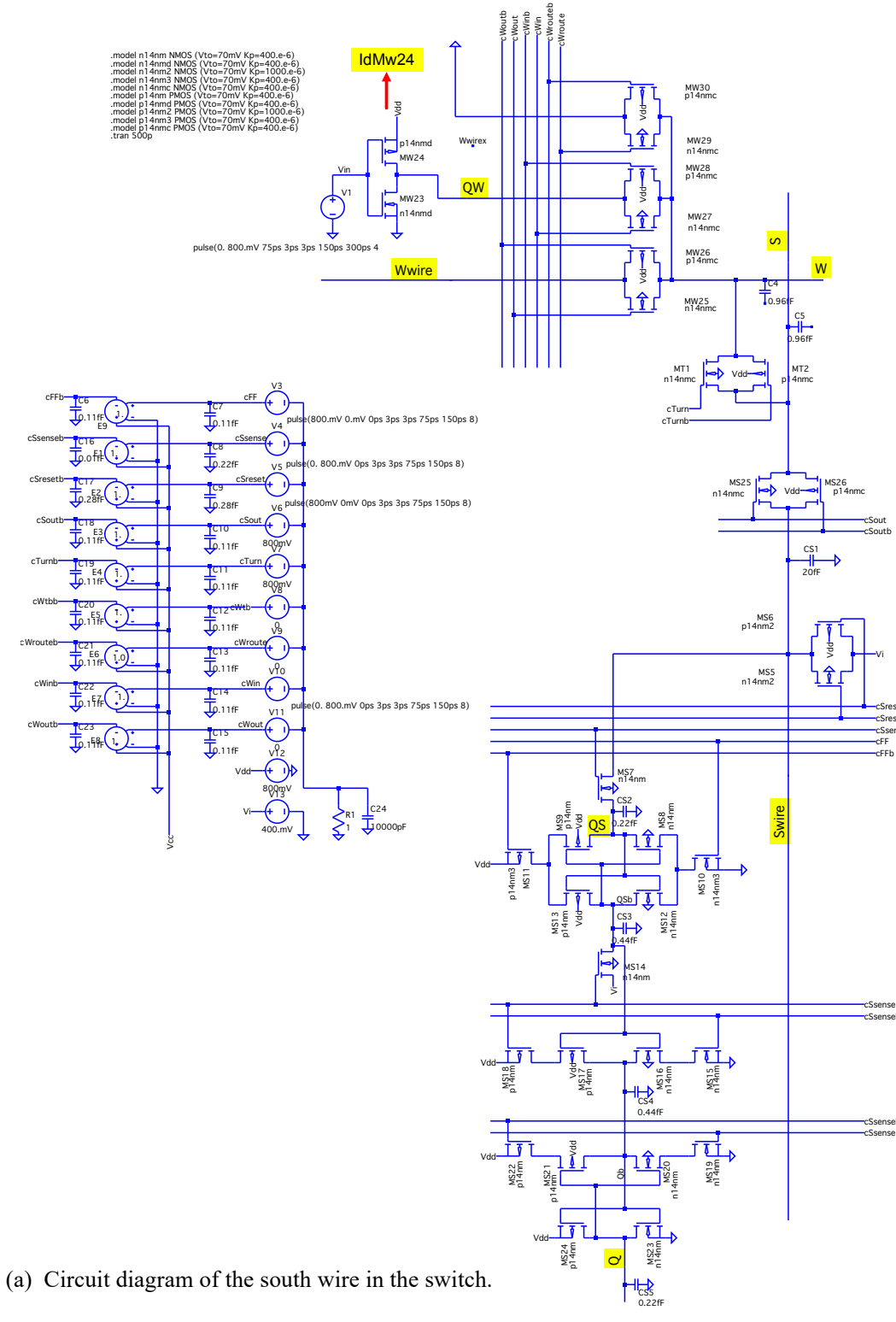

Fig. 13. Switch implementation.

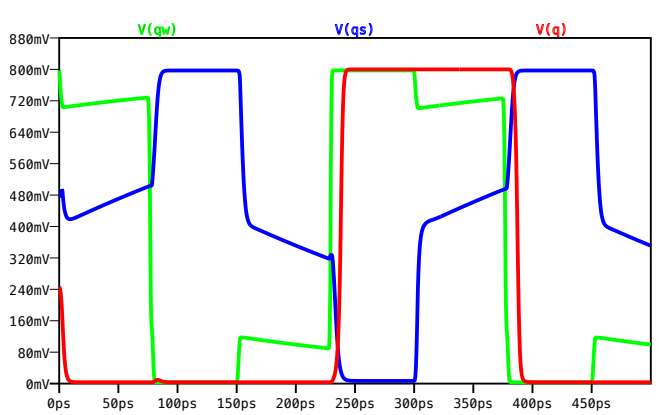

(b) Waveforms of $Q W, Q S$ and $Q$ in the switch.

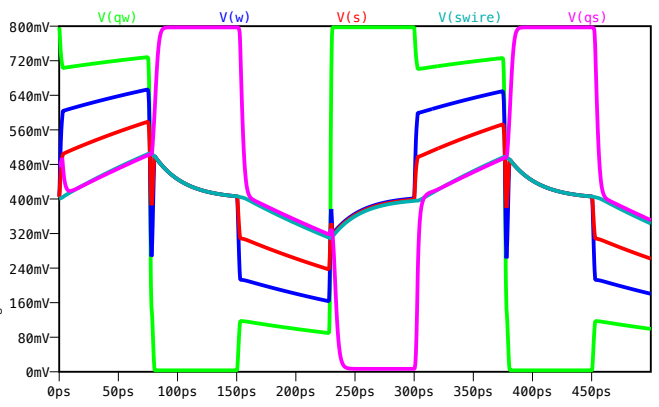

(c) Waveforms of $Q W, W, S$, Swire and $Q S$ in the switch.

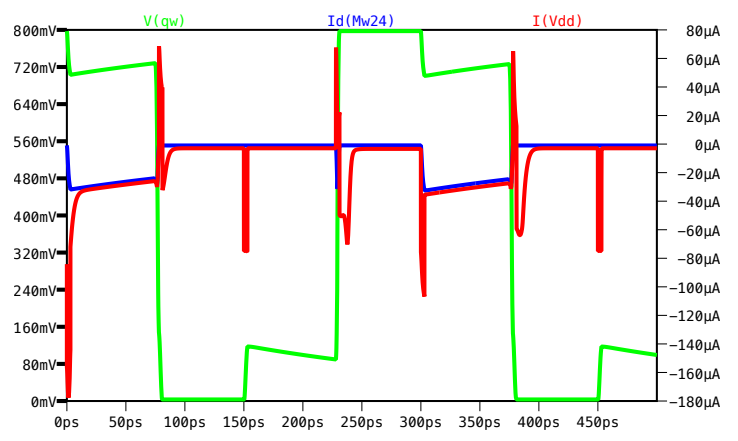

(d) Waveforms of $Q W$ together with $I d M w 24$ and $V_{D D}$ currents in the switch. 
scaling the transistors, the amplitudes would increase. However, the transistors become wider and their sizes are more than double that of the CMOS transistors. The control energy becomes considerably higher; therefore, the CMOS version is preferred. The energy consumption for a datapath is $4.1 \mathrm{fJ} / \mathrm{bit} / \mathrm{hop}$.

\section{B. Switch Layout}

The layout, see Fig. 14, follows the logic structure, see Fig. 10. There are transmission wires from each direction that continue to the central part. Transistors for transmissions gates interrupt them. In the central area, there are two wires in different layers, one in each $\mathrm{x}$ - and $\mathrm{y}$-direction. In the crossover, there is a transmission gate. These gates form a diagonal through the central part. The combined flip-flop and sense amplifier has a pitch equal to the wire pitch. The turn buffers are situated as a register between the central part and the sense amplifiers.

There is one template pipelined wire used in each direction, see Fig. 15. Five of them are used for the tags U (free/used), first, straight, and left/right, and the first bit of the 32-bit payload. They use three AND-gates to decode the tag. There are 31 of them without the AND-gates used by the rest of the payload. There is additional circuitry for the $\mathrm{U}_{\mathrm{n}}$ bit. It does not contain any drivers or gates.

The three $U_{n}$ from left, straight and right are included in the AND-gates in the $\mathrm{W}$ and $\mathrm{E}$ directions. Wires for them pass the central part. Only the straight $U_{n}$ is used for $E$ and $N$ directions.

The layout of a pipelined wire is shown in Fig. 15. The layout is sketched since complete design rules are not

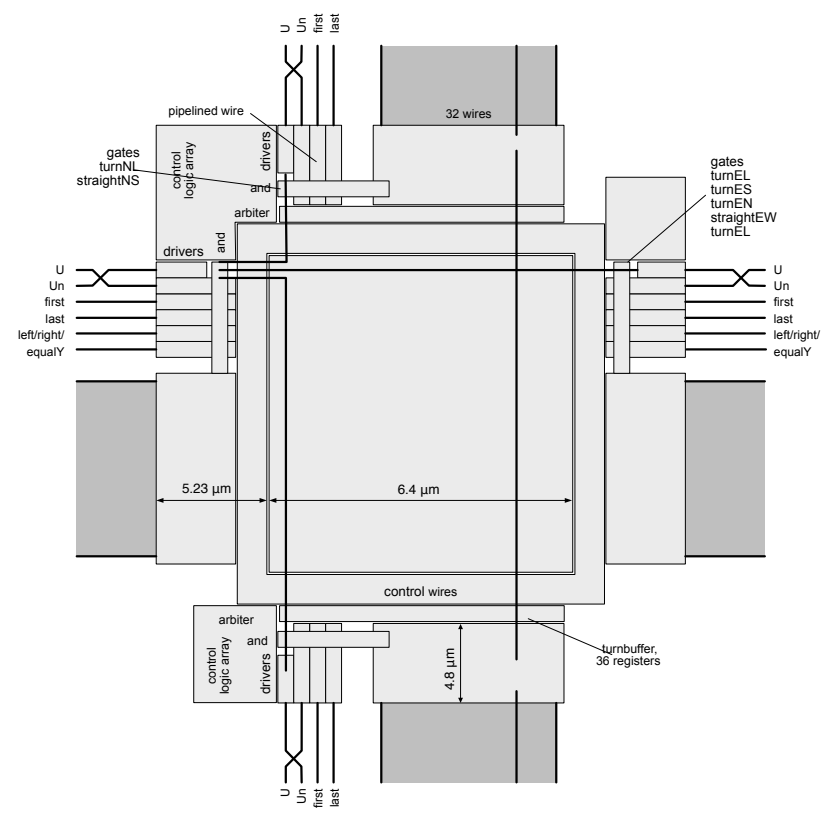

Fig. 14. Switch layout. available to us. However, they are estimated from a published SRAM cell. To the left, the xwire/ywire is entered in metal M4 and to the right is the $\mathrm{x} / \mathrm{y}$-intersection. Internally it is metal M4 but in the central part metal M3 and metal M4, respectively. Over each pipelined wire device, there are wires passing in metal M3. They consist of ground, $\mathrm{V}_{\mathrm{DD}}$ and control signals.

The control signals are driven from the side of the vector of pipelined registers. There are four signals used to control the drivers. Two additional control signals are used for the turn buffer in the $\mathrm{N}$ and $\mathrm{S}$ directions. The vector decodes three tag signals. All these wires are connected to a control unit implementing the arbitrator, which probably will be placed in the corner of the PE.

\section{RELATED WORK}

\section{A. Network-on-Chip}

Many research reports mention machines using NoCs in their introductions.

In 2001 Dally and Towles proposed a first NoC [12]. It used a 2D torus network. Next year, Kumar et al proposed a standard methodology [13]. For the first time, they used the acronym NoC for Network-on-Chip.

Intel's Teraflop is an 80-core processor connected by mesh with five input nodes [14]. The mesh delivers $1.6 \mathrm{~Tb} / \mathrm{s}$ using $62 \mathrm{~W}$, or $38.7 \mathrm{pJ} / \mathrm{b}$. $30 \%$ of the power is spent on the network.

The MIT RAW processor contains a $4 \times 4$ mesh network [15]. It consumes $7.1 \mathrm{~W}$, or $36 \%$ of the power [7].

IBM's Power4 used a crossbar to connect L2 cache banks [16]. Injection bandwidth was $640 \mathrm{~Gb} / \mathrm{s}$.

The IBM Cell processor used an interconnect based on four concentric rings [17]. Injection bandwidth was $1.7 \mathrm{~Tb} / \mathrm{s}$.

IBM Cyclops-64 (C64) is a petaflop supercomputer [18]. A 3D mesh network connects it, where each node is a C64 chip. Each chip contains 80 processors connected by a 7 -stage pipelined crossbar with $96 \times 96$ ports. The crossbar has an area of $27 \mathrm{~mm}^{2}$, which is only $6 \%$ of the $462 \mathrm{~mm}^{2}$ chip area.

Blue Gene supercomputers were based on Cyclops-64. The Blue Gene/Q is optimized for price/performance, energy efficiency, and reliability. They used the $360 \mathrm{~mm}^{2}$ CQC chip with $1.47 \mathrm{G}$ transistors in a $45 \mathrm{~nm}$ technology implementing 18 PEs. These are connected to a 22-port crossbar, 256 bits wide with an aggregate bandwidth of $3.3 \mathrm{~Tb} / \mathrm{s}$.

TRIPS was a joint effort between the University of Texas at Austin and IBM, Intel, and Sun Microsystems. It used a $2 \times 10$ mesh using four virtual channels performing wormhole routing with 128 bits wide links [19]. It had a peak bandwidth of $608 \mathrm{~Gb} / \mathrm{s}$ where the emphasis was on low latency. Injection bandwidth was $590 \mathrm{~Gb} / \mathrm{s}$. The net is mainly used to connect processors and L2-caches.

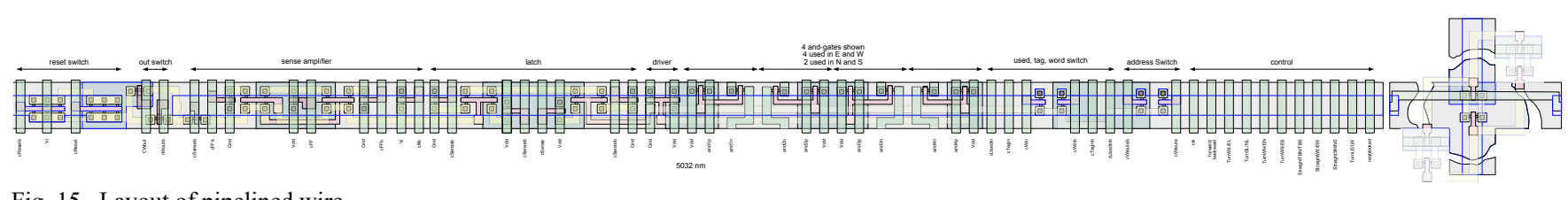

Fig. 15. Layout of pipelined wire. 
The Tilera Tile64 processor, that contains 64 core processors, uses five independent mesh networks with 32-bit links [20], [21], [22]. Each node has five duplex ports. The network supports $960 \mathrm{~Gb} / \mathrm{s}$.

In comparison, the Bubble NoC described in this article provides up to $15 \mathrm{~Gb} / \mathrm{s}$ full duplex transport capacity per $\mathrm{PE}$, taking the bubble flow control mechanism into account, and an SBP device may contain tens of thousands of PEs. The aggregate bandwith may therefore well exceed $150 \mathrm{~Tb} / \mathrm{s}$ (this is for 10,000 PEs).

\section{B. Detailed Comparison}

In this section, the performance and energy consumption of two state-of-the-art NoCs are compared to the Bubble NoC.

This means three kinds of processing elements of varying complexity and silicon area are considered. In a $14 \mathrm{~nm}$ technology, CISC cores are in the range of a few $\mathrm{mm}^{2}$, manycore processor (MCP) cores come down to around $0.1 \mathrm{~mm}^{2}$, and the SBP core approaches $0.01 \mathrm{~mm}^{2}$. A CISC is characterized by its large cache and achieves high speed by integrating a generous amount of transistors. An MCP is generally without cache, but it has a memory system that is partly shared between the PEs and is designed for high speed with limited memory requirements. The SBP is optimized for low energy and high speed within a limited area. This is achieved by a unified and integrated memory system with a density comparable to ordinary DRAMs.

No recent publications about NoCs that are particularly aimed for MCPs have been found. For NoCs accommodated to CISCs, there are recent publications, such as FastTrackNoC [23] and FreewayNoC [24] which are compared to several state-of-the-art NoCs and found to be superior. Their area and energy consumption are much higher than those of the Bubble $\mathrm{NoC}$, while the speeds are in the same order. The functionality in terms of features must of course also be considered to provide a fair comparison.

In order to achieve this, some conversions have to be made. A hop is an arbitrary distance. Assume that the devices contain cores with approximately square shape. Their sides are almost proportional to the technology node $d t n(e . g ., 14 \mathrm{~nm}, 28 \mathrm{~nm}$, etc.). The hop length is therefore normalized to $d t n$. Both FastTrackNoC and FreewayNoC use $28 \mathrm{~nm}$ technology, while the SBP aims for a $14 \mathrm{~nm}$ implementation.

\section{1) Wire Delays}

State-of-the-art for $14 \mathrm{~nm}$ VLSI technology is air-isolated wires with a capacitance of $0.15 \mathrm{fF} / \mu \mathrm{m}$ and a resistance of $2.7 \Omega / \mu \mathrm{m}$ [25], thereby providing a time constant of $0.405 \mathrm{fs} / \mu \mathrm{m}^{2}$. The delay for the $150 \mu \mathrm{m}$ wire in a Bubble NoC is $9.1 \mathrm{ps}$ and applied to FastTrackNoC with $2.1 \mathrm{~mm}$ [23] wires it is $1.8 \mathrm{~ns}$, and for FreewayNoC with $2.85 \mathrm{~mm}$ [24] wires it is $3.3 \mathrm{~ns}$ according to Spice. Some kind of sense amplifier is required to exceed the time constant. The Bubble NoC with a cycle time of $150 \mathrm{ps}$ has a good margin due to its short links. However, FastTrackNoC and FreewayNoC use cycle times 327 ps [23] and 340 ps [24]. This requires the wires to be separated into several segments, as was described in section II.D and illustrated in Fig. 4.

When using standard CMOS circuits, the propagation delays should match the transport frequency. This can be achieved by pipelining the wire. Bubble $\mathrm{NoC}$ has an appropriate cycle, while FastTrackNoC and FreewayNoC need 3 and 4 stages with link propagation delays of 980 ps and $1.72 \mathrm{~ns}$.

\section{2) Energy}

The energy that a wire requires depends mainly on its capacitance. When running faster than the wire time constant, the energy dissipation increases. The switching energy for one link capacitance with $0.8 \mathrm{~V}$ voltage swing is $14.4 \mathrm{fJ}$ for the Bubble NoC, $202 \mathrm{fJ}$ for the FastTrackNoC, and $274 \mathrm{fJ}$ for the FreewayNoC. The energy consumption per hop at a reasonably low load is $4.1 \mathrm{fJ}$ for the Bubble $\mathrm{NoC}, 1.5 \mathrm{pJ}$ for the FastTrackNoC and $2.3 \mathrm{pJ}$ for the FreewayNoC. The two last will halve when compensating for their $28 \mathrm{~nm}$ technology.

The energy consumption per bit divided by the capacitive wire energy is one way to measure of how efficiently the router is implemented. For Bubble NoC the relationship is 0.28 times, for FastTrackNoC 7.45 times, and for FreewayNoC 8.40 times.

When the device is a memory, a path passes a certain number of memory cells per time unit. The memory cell has a pitch proportional to the technology. A way to measure is the energy to pass a memory cell per technology, i.e., $14 \mathrm{~nm}, 28$ $\mathrm{nm}$ and $28 \mathrm{~nm}$ respectively, is for Bubble NoC $0.38 \mathrm{aJ}$, FastTrackNoC $20 \mathrm{fJ}$, and FreewayNoC $22 \mathrm{fJ}$. The last two have to be compensated for the technology by a factor of $1 / 2-1 / 4$ but are still very much higher.

Another way to measure is how well the power density matches the device. It is the product of hop frequency and energy per hop divided by the device area: Bubble NoC $3.9 \mathrm{~W} / \mathrm{cm}^{2}$, FastTrackNoC $26.7 \mathrm{~W} / \mathrm{cm}^{2}$, and FreewayNoC $16.9 \mathrm{~W} / \mathrm{cm}^{2}$. The power density on chips is generally much higher, thus the NoC power consumption is far from a major part of the processor.

\section{3) Speed}

The speed can be measured as the raw number of bits transferred per unit area: Bubble NoC $470 \mathrm{Tbit} / \mathrm{cm}^{2}$, FastTrackNoC 4.4 Tbit $/ \mathrm{cm}^{2}$, and FreewayNoC $1.8 \mathrm{Tbit} / \mathrm{cm}^{2}$. Here, the Bubble NoC really excels.

The latency can be expressed as the time delay per technology, i.e., $14 \mathrm{~nm}, 28 \mathrm{~nm}$ and $28 \mathrm{~nm}$ respectively: for Bubble NoC $18 \mathrm{Tbit} / \mathrm{s}$, FastTrackNoC $57 \mathrm{Tbit} / \mathrm{s}$ and FreewayNoC 39 Tbit/s. The main reason that the Bubble NoC is slower is that a single wire is used for duplex halving the capacity. The Bubble NoC is optimized to support far more parallel processes than the other architectures are designed for, which makes it much less susceptible to latency time.

A large system performs a vast number of accesses, where each global access uses a path. The system has a total path length which loads each link that is passed. Every individual link contributes with its own load. A single overloaded link 
may cause congestion and limit the system performance. A rather stable load could be achieved by rerouting, but the Bubble NoC does not support rerouting. Instead, it is based on the allocation of processes to be beneficial.

\section{4) Heavy Load}

Consider a transport along the $\mathrm{x}$-axis and assume there is an equal and low injection rate in each node. There is a possibility of an injected flit and a passing flit simultaneously. At low loads this possibility causes additional delays along the $\mathrm{x}$-axis, however very seldom. When the probability reaches above 30 percent the delay increases considerably, because the $\mathrm{x}$-pipeline becomes heavily loaded. In conventional networks, and especially in bufferless networks, the network load cannot be increased beyond a certain limit.

In a bubble controlled flow, every node may absorb a flit. All links may contain a flit, and there is always a movement forward. If every node injects flits, the pipeline will constantly be filled. Fairness at each node allows flits to pass from upstream, and at each node a certain fraction will pass. A long distance flit has an exponentially falling probability to reach a node further downstream, as in car traffic jams. Short distance flits will propagate with moderate speed. The situation is the same along the y-axis, however, here turns cause the injection of flits. An infinite system randomly injecting flits of length $m l$ with the same path length $d x+d y$ would have a speed given by the use of $m l \times(d x+d y)$ links. The mean absorption rate is finite and reduced to $1 /(m l \times(d x+d y))$ times the clock speed. The fairness problem has not yet been thoroughly studied by us, but there will be a graceful degradation compared to the hard limit in bufferless networks.

\section{5) Area}

The areas are not specified for FastTrackNoC and FreewayNoC, however, their gate counts, 178,652 and 276,732, correspond to about a million transistors. A Bubble NoC node has a transistor count in the order of 5,900.

The wire lanes are time-division duplexed in Bubble NoC but require separate nets in the others. The lane for Bubble NoC contains 38 wires, FastTrackNoC uses $2 \times 152$ wires and FreewayNoC $2 \times 159$ wires. The wire lane widths for FastTrackNoC and FreewayNoC are not known but can be expected to be around $100 \mu \mathrm{m}$ in $28 \mathrm{~nm}$ technology while the Bubble NoC only needs $9 \mu \mathrm{m}$ in $14 \mathrm{~nm}$ technology. They use 12 percent of the total area for Bubble NoC, 4.6 percent for FastTrackNoC, and 3.6 percent for FreewayNoC.

\section{Discussion}

The Bubble NoC is superior in all aspects except latency time, where it is somewhat slower. FastTrackNoC and FreewayNoC are intended for CISC networks and fit in well there. However, they are unsuitably big and complex for MCP and SBP devices.

How can the Bubble NoC be adapted to CISC? Duplex is already there, but the datapath may be increased from 32 to 128 payload pipelined wires. The wire lanes will be accordingly wider, and the drivers need to be four times larger. This can be achieved by using four transistors in parallel in the drivers and will increase the area slightly.

The corresponding link length is about $1 \mathrm{~mm}$. This would require seven simplified routers along the link for the pipelining. These routers only need straight NS and SN operations, so most of the control logic can be eliminated, but the bubbles must be handled. The latency time does not match the FastTrackNoC router, therefore the distance between the routers must be increased at the same clock frequency.

Because of this, two $550 \mu \mathrm{m}$ links may be used. The wire time constant is $122 \mathrm{ps}$, which allows for a clock of $150 \mathrm{ps}$. The injected current in the wires needs to be 3.7 times higher than described earlier, so the number of buffer transistors must increase from one to four. These transistors may be placed across the wire lanes. They would be parallel and add some few $\mu \mathrm{m}$ to the length of the pipelined wire. The control wires have to drive 512 transistors instead of 32 . These drivers are feasible and would increase the width of the drivers at the side of the pipeline datapath.

This design will have about the same access latency time and transport speed as FastTrackNoC and FreewayNoC, but narrower wire lanes, a much smaller router, lower energy consumption, and very simple control. The linear address reach would increase from 32 steps to 128 , which will be sufficient for most purposes. From a design point of view, such a Bubble NoC could be made available as a cell in a standard library.

\section{Conclusion}

Climate change predicts a global warming. However, the current trend in computing is an increasing use of power and energy. Emerging large data centers and AI on consumer level will further increase the energy consumption. These applications are large multiprocessor systems where communication is critical. The world has to reduce the energy use by an order of magnitude; however, this is not yet feasible. New types of low power multiprocessors have to emerge.

Speed and energy are equally important. The trend of CISC processors has been to add more transistors to increase speed and rely on hardware evolution to compensate for the energy requirements. Some other processors guide another trend by reducing complexity, cost and energy consumption. This work is one step towards such processing.

A PE uses energy for arithmetic and memory accesses. In both of them, long wires are the primary energy consumers. The physical size of emerging PEs has to be reduced to minimize energy consumption, especially in the memory. Communication between these small elements will increase, but the area portion for the network should be reduced.

The Bubble NoC is designed to match this. Its performance exceeds other contemporary NoCs, and it is also much smaller. It can be adapted to various link lengths and widths. The guaranteed delivery increases reliability and usefulness. 


\section{ACKNOWLEDGMENT}

The authors wish to thank Prof. Per Stenström at Chalmers University of Technology for his support.

\section{REFERENCES}

[1] M. Rimborg, P. Trancoso and G. Carlstedt, "PHOENIX: Efficient Computation in Memory," Proceedings of the International Symposium on Memory Systems, Alexandria, VA, October 2017, pp. 15-25, https://doi.org/10.1145/3132402.3132430.

[2] J.-Y. Fourniols, M. Roca, F. Caignet and E. Sicard, "Characterization of crosstalk noise in submicron CMOS integrated circuits: an experimental view," in IEEE Transactions on Electromagnetic Compatibility, vol. 40, no. 3, Aug. 1998 , pp. 271-280.

[3] A. Deutsch et al., "Modeling and characterization of long on-chip interconnections for high-performance microprocessors," in IBM Journal of Research and Development, vol. 39, no. 5, Sep. 1995, pp. 547-567.

[4] H. Zhang, V. George and J. M. Rabaey, "Low-swing on-chip signaling techniques: effectiveness and robustness," in IEEE Transactions on Very Large Scale Integration (VLSI) Systems, vol. 8, no. 3, June 2000, pp. 264-272.

[5] J. Duato, "A necessary and sufficient condition for deadlock-free routing in cut-through and store-and-forward networks," in IEEE Transactions on Parallel and Distributed Systems, vol. 7, no. 8, Aug. 1996, pp. 841854.

[6] Z. Lu, M. Zhong and A. Jantsch, "Evaluation of on-chip networks using deflection routing," Proceedings of the 16th ACM Great Lakes symposium on VLSI, Association for Computing Machinery, New York, NY, USA, 2006, pp. 296-301.

[7] Hangsheng Wang, Li-Shiuan Peh and S. Malik, "Power-driven design of router microarchitectures in on-chip networks," Proceedings. 36th Annual IEEE/ACM International Symposium on Microarchitecture, 2003. MICRO-36., San Diego, CA, USA, 2003, pp. 105-116.

[8] W. Dally and B. Towles, "Principles and Practices of Interconnection Networks," San Francisco, CA: Morgan Kaufmann Publishers Inc., 2003.

[9] C. Lin et al., "High performance 14nm SOI FinFET CMOS technology with $0.0174 \mu \mathrm{m}^{2}$ embedded DRAM and 15 levels of $\mathrm{Cu}$ metallization," 2014 IEEE International Electron Devices Meeting, San Francisco, CA, 2014, pp. 3.8.1-3.8.3.

[10] D. Ingerly et al., "Low-k interconnect stack with metal-insulator-metal capacitors for $22 \mathrm{~nm}$ high volume manufacturing," 2012 IEEE International Interconnect Technology Conference, San Jose, CA, 2012, pp. 1-3.

[11] S. Narasimha et al., "22nm High-performance SOI technology featuring dual-embedded stressors, Epi-Plate High-K deep-trench embedded DRAM and self-aligned Via 15LM BEOL," 2012 International Electron Devices Meeting, San Francisco, CA, 2012, pp. 3.3.1-3.3.4.
[12] W. J. Dally and B. Towles, "Route packets, not wires: on-chip interconnection networks," in Proceedings of the 38th annual Design Automation Conference (DAC '01). Association for Computing Machinery, New York, NY, 2001, pp. 684-689.

[13] S. Kumar et al., "A network on chip architecture and design methodology," Proceedings IEEE Computer Society Annual Symposium on VLSI. New Paradigms for VLSI Systems Design. ISVLSI 2002, Pittsburgh, PA, 2002, pp. 117-124.

[14] Y. Hoskote, S. Vangal, A. Singh, N. Borkar and S. Borkar, "A 5-GHz Mesh Interconnect for a Teraflops Processor," in IEEE Micro, vol. 27, no. 5, Sept.-Oct. 2007, pp. 51-61.

[15] M. B. Taylor et al., "The Raw microprocessor: a computational fabric for software circuits and general-purpose programs," in IEEE Micro, vol. 22, no. 2, March-April 2002, pp. 25-35.

[16] J. M. Tendler, J. S. Dodson, J. S. Fields, H. Le and B. Sinharoy, "POWER4 system microarchitecture," in IBM Journal of Research and Development, vol. 46, no. 1, Jan. 2002, pp. 5-25.

[17] D. C. Pham et al., "Overview of the architecture, circuit design, and physical implementation of a first-generation cell processor," in IEEE Journal of Solid-State Circuits, vol. 41, no. 1, Jan. 2006, pp. 179-196.

[18] Ying Ping Zhang, Taikyeong Jeong, Fei Chen, Haiping Wu, R. Nitzsche and G. R. Gao, "A study of the on-chip interconnection network for the IBM Cyclops64 multi-core architecture," Proceedings 20th IEEE International Parallel \& Distributed Processing Symposium, Rhodes Island, 2006, pp. 10.

[19] P. Gratz et al., "Implementation and Evaluation of On-Chip Network Architectures," 2006 International Conference on Computer Design, San Jose, CA, 2006, pp. 477-484.

[20] D. Wentzlaff et al., "On-Chip Interconnection Architecture of the Tile Processor," in IEEE Micro, vol. 27, no. 5, Sept.-Oct. 2007, pp. 15-31.

[21] "The Tile Processor ${ }^{\mathrm{TM}}$ architecture: Embedded multicore for networking and digital multimedia," 2007 IEEE Hot Chips 19 Symposium (HCS), Stanford, CA, 2007, pp. 1-12.

[22] S. Bell et al., "TILE64 - Processor: A 64-Core SoC with Mesh Interconnect," 2008 IEEE International Solid-State Circuits Conference - Digest of Technical Papers, San Francisco, CA, pp. 88-598, 2008.

[23] A. Ejaz and I. Sourdis. "FastTrackNoC: A DDR NoC with FastTrack Router Datapaths," 2021, https://research.chalmers.se/publication/522726/file/522726_Fulltext.pdf

[24] A. Ejaz, V. Papaefstathiou and I. Sourdis, "FreewayNoC: A DDR NoC with Pipeline Bypassing," 2018 Twelfth IEEE/ACM International Symposium on Networks-on-Chip (NOCS), 2018, pp. 1-8.

[25] K. Fischer et al., "Low-k interconnect stack with multi-layer air gap and tri-metal-insulator-metal capacitors for $14 \mathrm{~nm}$ high volume manufacturing," 2015 IEEE International Interconnect Technology Conference and 2015 IEEE Materials for Advanced Metallization Conference (IITC/MAM), 2015, pp. 5-8. 